\title{
A novel finding in MNGIE (Mitochondrial Neurogastrointestinal Encephalomyopathy): hypergonadotropic hypogonadism
}

\author{
İsmail Hakkı Kalkan, Öykü Tayfur, Erkin Öztaş, Yavuz Beyazit, Hakan Yildiz, Bilge Tunç
}

Gastroenterology, Türkiye Yuksek Ihtısas Hospital, Ankara, Turkey

Mitochondrial neurogastrointestinal encephalomyopathy (MNGIE) is a rare autosomal recessive multisystem disorder caused by mutations in the gene encoding thymidine phosphorylase (endothelial cell growth factor 1) (ECGF1). MNGIE is characterized by severe gastrointestinal dysmotility and leads to cachexia, ptosis, external ophthalmoplegia, peripheral neuropathy, and leukoencephalopathy. ${ }^{1}$ MNGIE is a rare disorder that can appear any time from childhood to adulthood. Its prevalence is unknown while marked delay in the diagnosis is common. ${ }^{2}$ Herein we report a unique case of MNGIE that has an endocrinologic involvement presenting with primary hypogonadism.

A 23-year old male with episodic vomiting and epigastric pain had been investigated for the last six years. He was cachectic (weight, 41kg; height, 160 $\mathrm{cm}, \mathrm{BMI}: 16.0 \mathrm{~kg} / \mathrm{m}^{2}$ ). He had generalized muscle weakness and atrophy, absent tendon reflexes in the lower extremities, mild bilateral ophthalmoplegia and ptosis, gynecomastia, hair loss in the pubic area, and subnormal testicular volume. Although he had

Key words: Hypergonadotropic hypogonadism, Mitochondrial myopathy, MNGIE

Address for correspondence:

İsmail Hakkı Kalkan, MD, Attar Sokak 21/14, Gaziosmanpaşa/ Çankaya 06700, Ankara, Turkey, Tel.: +90 50527040 85, e-mail: drismailster@gmail.com

Received 13-03-12, Revised 15-06-12, Accepted 02-07-12 a history of previous hospitalizations several times at various institutions, no specific disease had been identified. He was finally prescribed a therapeutic trial of gluten free regimen because of equivocal findings associated with celiac disease, but his symptoms did not disappear. He is a member of a consanguineous family with four children. The three brothers had suffered from similar symptoms and died.

Upper gastrointestinal endoscopy revealed esophagitis (Los Angeles Stage D) and pangastritis. Pathological examination of a duodenal biopsy specimen was not compatible with gluten sensitive enteropathy (GSE). Specific antibodies for GSE were also negative in the patient's serum. Direct radiography revealed air-fluid levels in the small intestinal segments and showed a hypotonic-ptotic stomach and diffuse thickening of the small intestinal wall following diluted barium ingestion. The mucosal folds could not be observed clearly, but the passage appeared to be normal on imaging. Endoscopic examination of the colon was normal.

Due to the patient's neurologic symptoms, an electromyography was performed. As a result, bilateral sensorimotor mixed type polyneuropathy was detected, especially in the lower extremities. Brain magnetic resonance imaging showed an increase in signal, affecting the subcortical and periventricular white matter of both hemispheres indicative of diffuse leucoencephalopathy. His muscle biopsy documented mitochondrial myopathy. Due to his 
physical findings that pointed to hypogonadism, FSH-LH and total testosterone levels were analyzed, the examination disclosing increased FSH level $(42.5 \mathrm{mIU} / \mathrm{ml}$, Normal Range: $0.7-11.1 \mathrm{mIU} /$ $\mathrm{ml})-\mathrm{LH}(25.5 \mathrm{pg} / \mathrm{ml}$, Normal Range: $0.8-7.6 \mathrm{mIU} / \mathrm{ml})$ and decreased total testosterone (41 ng/dl, Normal Range:181-1600 ng/dl) level. These findings further supported primary hypogonadism. MNGIE disorder was suspected because of the phenotypic manifestations, radiological findings, and also the family history. For the definitive diagnosis we performed genetic testing and sequencing of $E C G F 1$, which revealed homozygosity for a previously described splice-site mutation (IVS9-1G_A), confirming the diagnosis of MNGIE. ${ }^{3}$ After a three-week supportive treatment including antibiotics, intravenous fluids, and parenteral nutrition, his symptoms partially disappeared. Therefore, he was discharged and investigated further on an outpatient basis.

Clinically, MNGIE is observed between the 1st and the 5th decades, but the typical age of onset is in the second decade. Life expectancy is limited and death usually occurs during the third or fourth decades, generally due to cachexia and intestinal complications. ${ }^{2}$ Clinical appearance of MNGIE can be heterogeneous, but it usually is initiated by the symptoms of gastrointestinal dysmotility, such as diarrhea, vomiting, and intestinal pseudo-obstruction.

Clinical diagnostic criteria have been established for MNGIE. ${ }^{1}$ They include: (a) Severe gastrointestinal dysmotility manifesting as early satiety, abdominal cramps, and diarrhoea, or in the more severe cases as repeated attacks of vomiting and pseudoobstruction which can lead to laparotomies and the need for parenteral nutrition; (b) Cachexia following the above; (c) Ptosis; (d) External ophthalmoparesis; (f) Peripheral neuropathy; (g) Leucoencephalopathy. Other clinical manifestations are: hearing loss, areflexia, and proximal muscle. Our case completely fulfilled the clinical criteria of MNGIE with thymidine phosphorylase gene mutation. Currently, no definite treatment is available for MNGIE; however it is proposed that allogeneic stem cell transplantation may correct the biochemical derangements in such patients. There is also a recent report of the clinical benefit of peritoneal dialysis for gastrointestinal symptoms in a patient with MNGIE. ${ }^{2}$
Our patient was unique for the reason that he had hypergonadotropic hypogonadism. The only report in the literature that describes a case with MNGIE syndrome accompanying hypogonadism belongs to Artal FJ et al. ${ }^{2}$ However, while the latter patient had hypogonadotropic hypogonadism, our patient had hypergonadotropic hypogonadism. As Artal FJ states, it is unclear whether the changes observed in the central nervous system in MNGIE could account for this endocrine imbalance. Endocrinological abnormalities (including hypogonadism) related to the hypothalamo-pituitary axis and diabetes mellitus (DM) have been reported in mitochondrial genetic defects, indicating that these multisystemic diseases also involve various endocrine tissues. DM is thought to be due to a failure of energy production in the pancreatic B cells. A defect of mitochondrial oxidative phosphorylation may lead to the failure of hormone secretion in the hypothalamo-pituitary axis, which is an energy dependent process. Ischemic damage to endocrine organs due to mitochondrial abnormalities has been proposed as an alternative mechanism possibly triggering endocrinologic abnormalities. ${ }^{4}$ Hypogonadotropic hypogonadism has been reported in other mitochondrial encephalomyopathies, such as autosomal dominant progressive external ophthalmoplegia, which is a mitochondrial genetic disease like MNGIE. ${ }^{5}$ Quade A et al reported a patient with mitochondrial myopathy showing evidence of chronic progressive external ophthalmoplegia. This patient had hypergonadotropic hypogonadism, supporting endocrine tissue involvement in mitochondrial myopathies. ${ }^{6}$

Although generally hypogonadotropic hypogonadism can be observed in mitochondrial genetic defects due to impairment in hormone secretion of the hypothalamo-pituitary axis, our patient had hypergonadotropic hypogonadism. We believe that the increase of gonadotropic hormones in our patient was secondary to testicular dysfunction as an endocrine end-organ. Also, the testicular dysfunction was a result of impairment of energy production because of a mitochondrial genetic defect. To the best of our knowledge, this is the first report in the literature of a patient presenting with hypergonadotropic hypogonadism accompanying MNGIE syndrome, a case that we felt noteworthy to report. In our patient, hypergo- 
nadotropic hypogonadism was another consequence of mitochondrial genetic defects affecting endocrine tissues, an occurrence not previously reported.

\section{CONFLICT OF INTEREST/STUDY SUPPORT}

Guarantor of the article: Ismail Hakk KALKAN

Financial support: None

Potential competing interests: None

\section{REFERENCES}

1. Bariş Z, Eminoğlu T, Dalgiç B, Tümer L, Hasanoğlu A, 2010 Mitochondrial neurogastrointestinal encephalomyopathy (MNGIE): case report with a new mutation. Eur J Pediatr 169: 1375-1378.

2. Carod-Artal FJ, Herrero MD, Lara MC, et al, 2007 Cognitive dysfunction and hypogonadotrophic hypo- gonadism in a Brazilian patient with mitochondrial neurogastrointestinal encephalomyopathy and a novel ECGF1 mutation. Eur J Neurol 14: 581-585.

3. Nishino I, Spinazzola A, Hirano M, 1999 Thymidine phosphorylase gene mutations in MNGIE, a human mitochondrial disorder. Science 283: 689-692.

4. Joko T, Iwashige K, Hashimoto T, et al, 1997 A case of mitochondrial encephalomyopathy, lactic acidosis and stroke-like episodes associated with diabetes mellitus and hypothalamo-pituitary dysfunction. Endocr J 44: 805-809.

5. Barrientos A, Casademont J, Genis D, et al, 1997 Sporadic heteroplasmic single $5.5 \mathrm{~kb}$ mitochondrial DNA deletion associated with cerebellar ataxia, hypogonadotropic hypogonadism, choroidal dystrophy, and mitochondrial respiratory chain complex I deficiency. Hum Mutat 10: 212-216.

6. Quade A, Zierz S, Klingmüller D, 1992 Endocrine abnormalities in mitochondrial myopathy with external ophthalmoplegia. Clin Investig 70: 396-402. 